\title{
Use of Model Predictive Control and Artificial Neural Networks to Optimize the Ultrasonic Release of a Model Drug From Liposomes
}

\begin{abstract}
Hesham G. Moussa, Member,IEEE, Ghaleb A. Huss Abstract-The use of echogenic liposomes to deliver chemotherapeutic agents for cancer treatment has gained wide recognition in the last 20 years. Cancerous cells can develop multiple drug resistance (MDR), in part, due to the drop in concentration of chemotherapeutic agents below the therapeutic levels inside the tumor. This suggests that MDR can be reduced by controlling the level of drug release in the diseased area. In this paper, a model predictive controller based on neural networks is proposed to maintain a constant chemotherapeutic release at the cancer site. The proposed system was able to follow the set point by varying the U.S. intensity within preset constraints. The system simulated model is viable and it showed a high average fit when stimulated with variable input variations, indicating the robustness of the nonlinear model. By maintaining a constant release of the drug so that the concentration level is above a certain threshold, we hope to reduce cancer resistance towards chemotherapeutic agents.
\end{abstract}

IndexTerms-Model predictive control(MPC), drug delivery, echogenic liposomes, neural networks (NN), NNMPC.

\section{INTRODUCTION}

C ANCER is a devastating disease that affects the lives of millions of patients worldwide. One of the mostly utilized medical treatments for this life-threatening ailment is chemotherapy, which involves the administration of therapeutic drugs that cause the death of malignant cells and tissues. However, chemotherapy is usually associated with multiple adverse side effects caused by the lack of specificity, as the drug does not differentiate between healthy and cancerous tissues [1]. Recently, efforts have been directed towards finding

Manuscript received December 18, 2015; revised April 26, 2016, July 3, 2016, September 11, 2016, and November 17, 2016; accepted December 28, 2016. Date of publication January 30, 2017; date of current version April 27, 2017. This work was supported by the American University of Sharjah Faculty Research Grant 1 under Gran AUS/FRG12012, and in part by the Patients Friends CommitteeSharjah, Al Qasimi Foundation, and AlJalila Foundation. Asteriskindicatescorresponding author.

H.G. Moussa is with the Department of Electrical and Computer Engineering, Waterloo University, Waterloo, ON N2L 3G1, Canada (email: hesham30moussa@gmail.com.

$*$ G. A. Husseini is with the Department of Chemical Engineering, American University of Sharjah, Sharjah 26666, United Arab Emirates (e-mail: ghusseini@aus.edu).

N. Abel-Jabbar is with the Department of Chemical Engineering, American University of Sharjah, Sharjah 26666, United Arab Emirates (e-mail: nabdeljabbar@aus.edu).

S. E. Ahmad is with the Center of NanoTechnology, University of Khartoum, Khartoum 11115, Sudan (e-mail: salma.e.a@gmail.com).

Digital Object Identifier 10.1109/TNB.2017.2661322 a solution to the non-specificity problem of anti-neoplastic agents. Encapsulating chemotherapeutic agents inside nanocarriers before injecting them into the patients body have shown promise in reducing these side effects. Some examples of these nanocarriers are micelles, dendrimers, archaeosomes, solid nanoparticles, and liposomes [2]. These carriers represent drug delivery systems (DDSs) that minimize the circulation of free drug in the body, which in turn reduces its interaction with healthy cells. The nanocapsules can circulate in the blood for an extended period of time and, with the help of various targeting techniques, the drug can accumulate preferentially at the tumor site. Once at the desired location, the drug release can be induced by external or internal means [3]. Liposomes and micelles are two of the most widely researched carriers in the field of drug delivery. They can be actively targeted using external (including light, temperature and ultrasound) or internal means (including $\mathrm{pH}$, enzyme and ligand targeting) [4]. Since Liposomes are FDA approved in cancer treatment since 1995, we opted to use these nanocarriers in this work. Liposomes are spherical nanovehicles made up of a phospholipid bi-layer capable of encapsulating hydrophobic drugs in their shell and hydrophilic drugs within their core. They are small in size (100-500 $\mathrm{nm}$ in diameter) and are capable of diffusing through blood capillaries into the tumor. Their physical characteristics, including their semi-solid nature, make them vulnerable to mechanical waves of certain frequencies; hence, their sensitivity to ultrasound [5], [6], [10]. What makes liposomes more appealing, for drug delivery applications, is their composition. Constructed using naturally occurring lipids, they have a structure resembling the human cell membrane, rendering them both stable and non-toxic when injected in the patient body. Nonetheless, without modifying their surface, the carriers are easily detected by the reticuloendothelial system and classified as foreign invaders leading to their opsonization and clearance by the liver [6]. To overcome this shortcoming, liposomes are chemically modified, using polyethylene glycol (PEG), to enhance their circulation time, and hence their ability to target cancer cells while sparing healthy cells. The liposomes used in this work are designed to be echogenic; i.e. activated by ultrasound. This type of liposomes is known as acoustically activated liposomes (AAL) [4], [7]. In addition to echogenicity, these liposomes are also designed to be stealthy, meaning that they can circumvent the body's defense mechanisms. Their radius range between 12.5 and $150 \mathrm{~nm}$ allowing them to utilize the enhance permeability and retention effect (EPR), also known as passive targeting, to accumulate preferentially at the tumor site [8]. The EPR effect ensures the preferential accumulation of drugloaded nanocarriers at the tumor site due 
to the leaky nature of blood capillaries found in tumors, which in turn allows liposomes to get trapped in those capillaries and eventually diffuse into the cancer tissue [9]. Once enough time has passed (to allow for the carriers extravasation at the tumor site), ultrasound is focused on the tumor to induce the temporal and spatial release of the anti-neoplastic agent to the diseased tissue. To achieve optimal release, the proper parameters (including ultrasonic frequency, power density and pulse duration) have to be optimized. A comprehensive survey on triggered drug release from liposomes can be found in [10]. An important factor that is critical to the effectiveness of the chemotherapy treatment is the drug concentration level. In order to guarantee a successful treatment and to reduce the chance of developing multi-drug resistance (MDR), the drug concentration has to be kept above a therapeutic level. Hence, when using a combination of liposomes and ultrasound as a DDS, it is advantageous to have a mechanism by which the drug concentration at the diseased site is carefully monitored and controlled (i.e.; if the drug level drops below the therapeutic window, more release can be induced and vice versa). In the present work, the level of release can be readily controlled by controlling the acoustic parameters employed. A model predictive controller (MPC) is used to develop a control system that is capable of controlling the release rate of a model drug (i.e. calcein) from liposomes by controlling the acoustic parameters employed. By measuring the concentration level and feeding it to the controller as a feedback signal, the desired drug concentration can be maintained. Since experiments were conducted under in vitro conditions with no cancer cells under treatment, drug concentration level was translated into percentage release from the liposomes. The MPC model presented herein is developed using in vitro drug release data using multiple input-output curves from which an approximate model was extracted [11]-[15]. The paper is organized as follows. Section 2 lists the methodology used to synthesize the loaded liposomes as well as provide some background on US as a drug release modality. Section 3 details the experimental setup used to collect the input-output curves used to design the system model. Section 4 discusses the proposed system. Section 5 presents background information on neural networks, followed by a discussion of the results and a brief conclusion in section 6 .

\section{MATERIALS AND Methods}

\section{A.Synthesis of PEGylated Liposomes}

The liposomes used in this work, which were designed to be passively targeted (by ensuring that their radius is below 250 nm) and acoustically activated, are PEGylated liposomes. To prepare these carriers, an amphiphilic PEG derivative group (para-nitrophenylcarbonyl-PEG-paranitrophenylcarbonyl;

(pNP-PEG-pNP)) is used to prepare DOPE (1,2-dioleoyl-snglycero-3-phosphoethanolamine)-

PEG-pNP conjugates which are then mixed with a lipidcholesterol solution. The liposomal bilayer shell is made up of three chemicals. The first is the 1,2-dipalmitoyl-snglycero-3phosphocholine (DPPC). Phosphatidylcholine (PC) lipids contribute, along with DOPE, to the ability of liposomes to encapsulate hydrophobic and hydrophilic drugs [16]. Cholesterol is also an ingredient in liposomal formation and is used to enhance the stability of the liposomes by restraining the presence of liquids. Cholesterol also minimizes the permeability of the liposomal membrane, accordingly, making it less leaky and having lower rates of lipid exchange with other structures in the blood circulation (such as red blood cells and lipoproteins). Most importantly, cholesterol effectively reduces drug bio-distribution and facilitates the elimination of liposomal residues after release [17], [18]. The third component used for liposomal formation is the DOPE-PEG-pNP conjugate. DOPE is one of the phospholipids that belong to the phosphatidylethanolamine (PE) family. Similar to PC and cholesterol, PE lipids are also naturally found in biological membranes, and the ratio of the $\mathrm{PC}$ to $\mathrm{PE}$ lipids is responsible for regulating the integrity of the membrane [19]. PEG chains act as spacer arms and are responsible for the stealthy nature of the liposomes, as it repels macrophages, decreases opsonization and increases the circulation time of these nanoparticles in the body. The procedure used to make the liposomes, based on a protocol by Torchilin and co-workers [5], is as follows: pNPPEG-pNP group is synthesized first, by reacting PEG2000-diol (HO-PEG-OH) with two molar equivalents of 4-nitrophenyl chloroformate (p-NPC) (Sigma-Aldrich Chemie $\mathrm{GmbH}$, Munich, Germany) in the presence of dichloromethane and pyridine (Scharlau Chemie, Barcelona, Spain) in a flask placed in an ice bath (p-NPC solution added drop wise under argon). The reactants are then allowed to react for 20 hours at room temperature with continuous stirring. Finally, pNP-PEG-pNP is precipitated using cold diethyl ether and dried overnight in a desiccator. This product is then reacted (in a round bottom flask) with one molar equivalent of DOPE (Sigma-Aldrich Chemie GmbH, Munich, Germany or Avanti Polar Lipids, Inc., Alabaster, AL, USA), both reactants are dissolved in dry chloroform with continuous stirring, in the presence of triethylamine (TEA). The reactants are allowed to react overnight at room temperature under argon to form the DOPEPEG-pNP conjugates. Chloroform is then evaporated in a rotary evaporator. The lipid residue is hydrated with $2 \mathrm{ml}$ of a $0.01 \mathrm{M}$ $\mathrm{HCl}-0.15 \mathrm{M} \mathrm{NaCl}$ solution and sonicated at in $40-\mathrm{kHz}$ sonicating bath (Elma D-78224, Melrose Park, IL, USA) for 10 minutes. The solution is then purified to separate the micelles formed during the sonication process from the unbound PEG and released pNP, using a Sephadex G-25 PD-10 desalting column (GE Healthcare Life Sciences, Pittsburgh, PA, USA). Finally, the solution is evaporated for 2 hours at high speed under vacuum in a rotary evaporator, and the DOPE-PEG-pNP is extracted 4 times with chloroform. The salt residues are precipitated on ice and removed by centrifugation. The produced DOPE-PEG-pNP is stored at $-20^{\circ} \mathrm{C}$ as a chloroform solution, with a concentration of $8.4 \mathrm{mM}$. DPPC (SigmaAldrich Chemie GmbH, Munich, Germany), cholesterol (AlfaAesar, Ward Hill, MA, USA), and DOPE-PEG-pNP are all dissolved in $2 \mathrm{ml}$ of chloroform, using a molar ratio of 68:30:2 (DPPC:Chol:DOPE-PEG-pNP). Chloroform is then evaporated under vacuum in a rotary evaporator to allow a lipidic film to form on the walls of the flask. The film is 
hydrated with a solution of calcein (Sigma-Aldrich Chemie $\mathrm{GmbH}$, Munich, Germany) at a self-quenching concentration (i.e. $30 \mathrm{mM}$ in a phosphatebuffered saline (PBS) buffer), with the $\mathrm{pH}$ adjusted to 7.4. The resulting solution is sonicated at maximum power using $40-\mathrm{kHz}$ ultrasound for $15 \mathrm{~min}$. The sample is then extruded three times $(10 \mathrm{x})$ through $0.45 \mu \mathrm{m}$ polycarbonate filters using an Avanti mini-extruder (Avanti Polar Lipids, Inc., Alabaster, AL, USA). The liposomes are cleaned using Sephadex G-25 PD-10 desalting columns (GE Healthcare Life Sciences, Pittsburgh, PA, USA) and suspended in PBS before being stored at $4^{\circ} \mathrm{C}$ until use. Our samples were characterized using a particle sizer. The results show that the synthesized liposomes are spherical in shape with a diameter ranging from $180 \mathrm{~nm}$ to $230 \mathrm{~nm}$. More details on characterizations can be found in [5] and [42].

\section{B.Ultrasound}

Ultrasound is widely researched as an effective triggering modality for drug release from echogenic nanoparticles [20]. US waves can be generated at different frequencies, intensities, duty cycles, etc. [21]. Echogenic liposomes are designed based on these parameters in order to obtain the desired drug release profile. There are four possible mechanisms that cause release from liposomes based on the US parameters used. The first mechanism is hyperthermia, which is usually associated with high intensity focused ultrasound (HIFU) at frequencies higher than $0.5 \mathrm{MHz}$ [22]-[27]. The second mechanism is capable of inducing release through the formation of microbubbles that undergo collapse cavitation events, the latter producing microjets and shockwaves sufficient to disturb the liposomal membrane, leading to the release of the encapsulated agent [28]-[30]. The third mechanism arises from the mechanical nature of US waves, the latter causing the liposomes to accelerate and collide with each other and/or their surroundings, leading in some instances to deformities in their membrane structures and allowing the encapsulated drug to diffuse out [31]. The fourth mechanism is the mechanism behind drug release from emulsion-liposomes (eLiposomes), which are the newest generation of echogenic liposomes that contain lowboiling point nanoemulsions [32]-[34]. In this work, low frequency ultrasound is used (at $20 \mathrm{kHz}$ ), hence the main physical mechanism behind the model drug release is acoustic cavitation; however, since the experiments are done in cuvettes, mechanical tiring due to collisions might have also contributed to the release [10]. In this work, a 3-mm probe connected to a VCX 750 actuator (Vibra cells, Sonics and Material) is used to trigger release from liposomes. The probe is tapered and produces an ultrasonic beam with a frequency of $20 \mathrm{kHz}$. The probe tip is water-resistant and is inserted into the solution in preparation for sonication. The solution is placed in a cuvette with an opening of dimensions $1 \mathrm{~cm} \times 1 \mathrm{~cm}$. This allows for an ample space for the probe to vibrate freely ensuring maximum energy transfer into the solution.

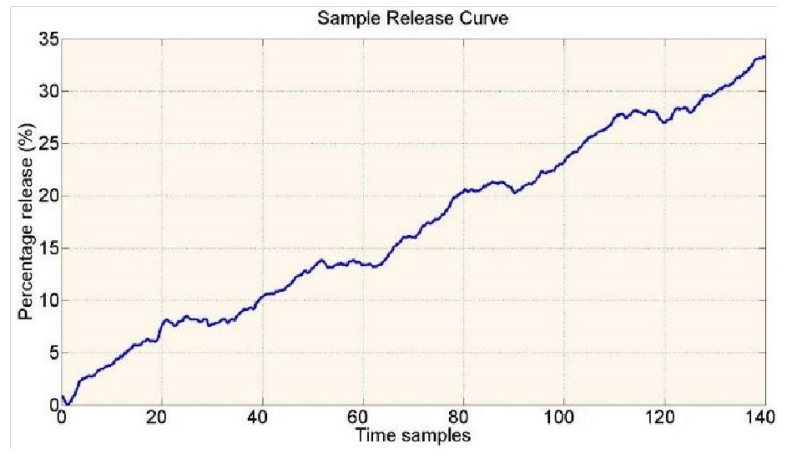

Fig. 1. Release percentage of calcein from liposomes.

\section{EXPERIMENTAL SETUP}

The experimental setup is as follows. An aliquot of the liposomal sample is first diluted in a cuvette using PBS (75 1 liposomes diluted with $2 \mathrm{ml}$ PBS). The cuvette is then placed in a QuantaMaster 30 Fluorescence Spectrofluorimeter (Photon Technology International, Birmingham, NJ, USA), which is used to measure the initial fluorescence level that corresponds to the baseline (F0), with the excitation and emission wavelengths of calcein set at $494 \mathrm{~nm}$ and $515 \mathrm{~nm}$, respectively, and a sampling rate of 10 points/seconds. This baseline is then used to normalize the collected data as will be discussed next. It is worth noting that the used liposomes showed no release in the absence of ultrasound. This result confirms previously published results showing zero drug release from liposomes when US is not applied [40]. The sample is then subjected to $20-\mathrm{kHz}$ US generated using a $3-\mathrm{mm}$ probe connected to a VCX 750 actuator. Three US powers were used $(6.09,7.8$, and 11.7 W). Since high intensities of ultrasound were applied, and to avoid over heating the sample, pulsed US was used rather than continuous wave US. The pulsed behavior was controlled by adjusting the duration of the ON and OFF periods to one of 2 settings: $50 \%$ ON cycle and $50 \%$ OFF cycle or $66 \%$ ON cycle and $34 \%$ OFF cycle. A slight temperature increase $\left(<2^{\circ} \mathrm{C}\right)$ was measured at $11.7 \mathrm{~W}$. It is important to note here that no statistically significant release was measured when the sample was heated to $42^{\circ} \mathrm{C}$ using a thermostatic bath (in the absence of ultrasound). Hence, we concluded that the acoustic release of calcein from liposomes was not due to hyperthermia but due to other effects (i.e. mechanical effects). The samples were sonicated for 5 minutes. A full pulsed ON-OFF cycle is 30 seconds during which the fluorescence level is continuously recorded. Figure 1 shows a sample curve of the results. The graph captures the release dynamics versus time. There is an evident increase in the fluorescence during the $\mathrm{ON}$ cycle of the ultrasound, while the release level plateaus when the US is switched off. The sonication is repeated at each setting 3 times and the average release calculated. At the end of each sonication cycle, the detergent Tx100 is added to each sample, to a final concentration of $0.48 \mathrm{mM}$, to lyse the remaining liposomes, allowing for the determination of a fluorescence level that represents $100 \%$ release. The recorded curves corresponding to 
the fluorescence level versus time are then converted to percent release curves versus time using the

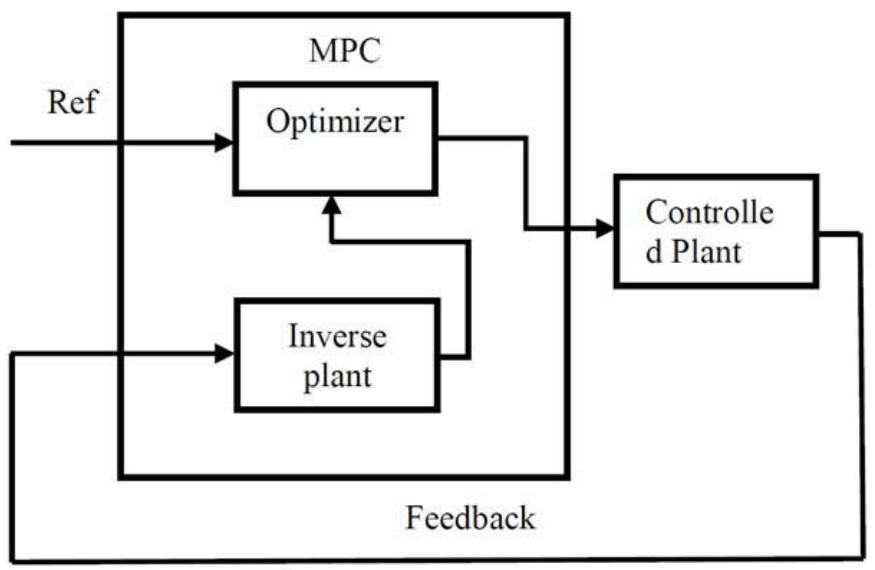

Fig. 2. Block diagram of the MPC.

equation below.

$\%$ Drugrelease $=\frac{F_{t}-F_{0}}{F_{\max }-F_{0}} * 100 \%$

where, $F_{t}=$ the instantaneous fluorescence level after subjecting the sample to $t$ insonation time; $F_{0}=$ initial fluorescence before insonation (baseline); $F_{\max }=$ maximum fluorescence intensity after adding detergent Tx100.

The main drawback associated with chemotherapy treatment is the development of MDR. The level of the chemotherapeutic agents present at the tumor site should be kept at or above a certain therapeutic level to reduce the chance of developing drug resistance; hence, it is critical to keep the drug concentration at or above these therapeutic levels [41]. To do so, the following system, described in section 4 , is proposed utilizing a neural network model predictive controller (NNMPC).

\section{NN-MPC (PROPOSED SYSTEM)}

The objective of the proposed system is to control drug release from liposomes using ultrasound. The system can be thought of as a single-input single-output system, where the ultrasonic pattern is the input while drug concentration level is the output. The system is a closed-loop control system with feedback that propagates information about current drug release level back to the controller as an error signal. In this work, we use a NN-MPC based control system to monitor and control drug levels by controlling the release rate from liposomes. The proposed system block diagram is shown in Figure 2.

In the context of drug delivery, a tumor site that is injected with liposomes filled with a chemotherapeutic drug can be thought of as the system under control and will be referred to as the liposomal drug delivery system for the rest of this work. The liposomal drug delivery system is subjected to US pattern to cause drug release from the liposomes. As the drug is released, the drug concentration level is measured and propagated back to the predictor. The predictor is a block that captures the inverse behavior of the body, i.e. it takes in drug release level as its input and produced US pattern as its output. Consequently, at any point in time, by creating an accurate model and uploading its inverse into the controller,

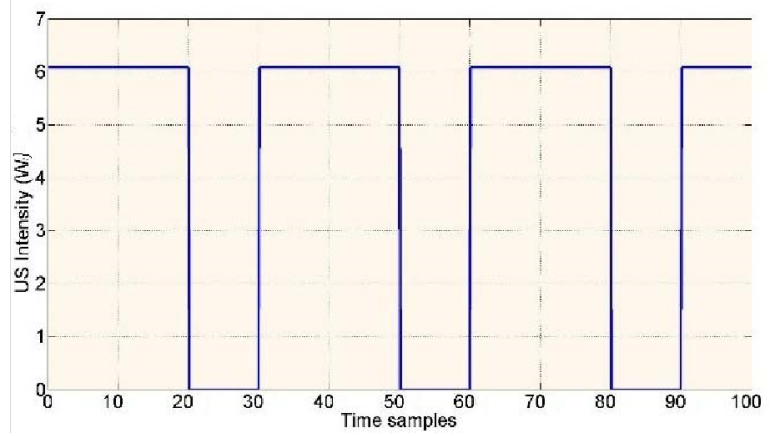

Fig. 3. Sample of the Input signal where the US power is set to $6 \mathrm{~W}$ and the ON cycle to 20 seconds, while the OFF cycle is 10 seconds.

the proposed MPC is assumed to be capable of predicting the utmost matched US parameters that incorporates the effect of the medium (the human tissues) on the wave, while efficiently minimizing the error between the set point and the current concentration level. As shown in Figure 2, an MPC is made up of three main blocks; namely: the controlled plant (liposomal drug delivery system), the inverse plant (predictor), and the optimizer [35]. The liposomal drug delivery model and the inverse model for the predictor were simulated using in vitro release data. The experiments utilized a cuvette filled with 30 $\mathrm{mM}$ of the liposomal solution in $2 \mathrm{ml}$ of PBS and a $20-\mathrm{kHz}$ probe to measure the acoustic release of a model drug from liposomes. US probe was inserted directly into the solution to cause drug release. Since chemotherapeutic drugs, such as DOX, are intrinsically fluorescent, the higher the measured fluorescence level, the higher the drug concentration level. Therefore, by recording the fluorescence level of the sample as it is being insonated, an estimate of the release percent can be measured. Multiple experiments were conducted using different US patterns, each had a different intensity and duty cycle, and liposomal concentrations. A large database of inputoutput curves was collected and used to develop a model that closely captures the behavior of the liposomes under different acoustic trigger signals. The model is used as an approximation for the liposomal drug delivery system in the proposed MPC [5], [42]. Since only in vitro release data were used in this work, the model can be further enhanced if in vivo experimental data are available which would allow us to include other parameters including cellular uptake to achieve a more realistic model. The output curves were then switched with the inputs and were used to estimate an inverse model that was used as the predictor. Various US patterns were used. Figure 3 shows an example of an acoustic input. To increase the accuracy of the estimated models, US patterns used had intensities ranging from $6.07 \mathrm{~W}$ to $11.7 \mathrm{~W}$. Also, the $\mathrm{ON}$ and OFF durations were varied. As is discussed earlier, artificial neural networks are the modeling technique of choice used to estimate the models. 
However, due to possible outliers within the data, numerous models were estimated from multiple input-output curves combination, and an average model that generates the best response to all inputs was derived. The same technique was used to approximate the inverse model; however, some difficulties were faced as the estimated models were unstable

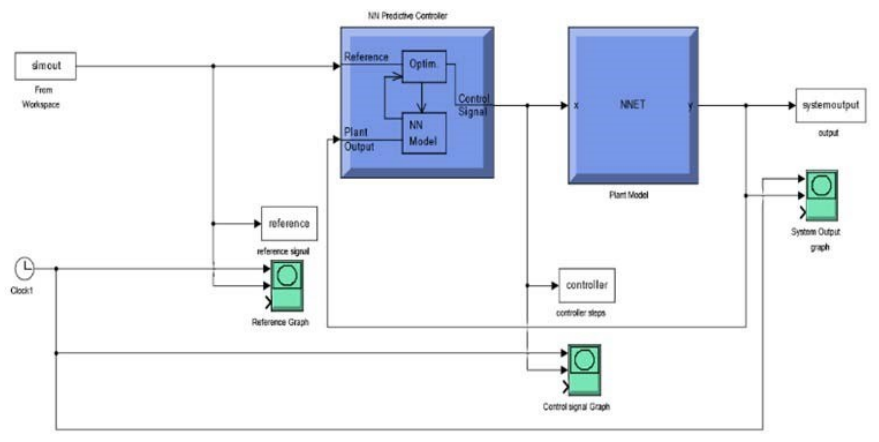

Fig. 4. SIMULINK generated schematic of the proposed/ simulated system.

due to fast changing edges in the output (the US pattern). Therefore, an equalization method was used to compensate for this behavior as is explained in the results section. As for the optimizer, a built-in MATLAB block is used (with minor parameter manipulation) to achieve optimal performance. The final system is built using SIMULINK; as shown in Figure 4. The function of the optimizer is to adjust the predicted US parameters produced by the inverse model based on the input reference signal. Essentially, an optimizer predicts several controller inputs (referred to as the control horizon), yet only the first predicted controller move is implemented. Based on these predictions, the calculated US input based on the inverse plant model is refined and passed on to the plant model. The corresponding output is then calculated and propagated back to the MPC, and the process is repeated until the reference curve is completely exhausted.

\section{BAckground on NeURAL NetWorks Modeling}

MPCs were first thought to work best with linear models; however, with the new advancements in modeling techniques, the performance of nonlinear-modeling MPCs was enhanced significantly proving otherwise. In this work, Artificial Neural Networks as a nonlinear modeling technique was used to find the plant model from the collected empirical data [36], [37]. There are many types of ANNs based on their topology, with feedforward neural networks (FFNNs) being the most widely used and are the topology of choice in this work [38], [39]. In this work, there are two applications in which a FFNN is used. The first is to perform plant identification and modeling using empirical data. The plant is portrayed as a black box, the model of which is predicted using FFNN. Second, FFNNs are used to build the MPC controller by finding an inverse plant model. For the first application, the plant model is estimated using experimental data. The first stage is the training stage. As is the case with any system identification, the system at hand is dealt with as a box that has input and output ports. To identify the system transfer function, input ports are excited with a known input signal and the output is captured. By finding the output over the input equation, the transfer function of the box is identified. Same procedure is followed using NNs to perform system identification. First, the physical system, cuvette with the diluted liposomal solution, is subjected to a known input (the input is US in this work),

TABLE I

Comparison Between the Performances of the Two DeVeloped MODELS AGAINST VARIOUS INPUTS

\begin{tabular}{|c|c|c|c|}
\hline Input curve & $\begin{array}{c}\text { Average } \\
\text { model 1 }(\%)\end{array}$ & $\begin{array}{c}\text { Average } \\
\text { model 2 (\%) }\end{array}$ & $\begin{array}{c}\text { Average } \\
\text { Inversre model (\%) }\end{array}$ \\
\hline Input 1 & 86.09 & 65.45 & 56.75 \\
\hline Input 2 & 97.39 & 92.47 & 74.92 \\
\hline Input 3 & 86.57 & 71.80 & 84.24 \\
\hline Input 4 & 6.69 & 77.43 & 76.43 \\
\hline Input 5 & 91.67 & 92.73 & 87.44 \\
\hline Input 6 & 93.41 & 66.63 & 69.57 \\
\hline Input 7 & 89.12 & 3.88 & 39.19 \\
\hline Input 8 & 86.84 & 89.06 & 81.68 \\
\hline Input 9 & 61.52 & 80.14 & 78.95 \\
\hline
\end{tabular}

then the corresponding output (percentage release drug) is captured. After that, the input and output data are used to train the NN. It is worth mentioning that the higher the complexity in the system being identified, the more data needed to perform system identification. Although, this training process might be enough for most applications, a further training step called open loop training is usually utilized to assert the accuracy of the model compared to the actual system. As for the second application of FFNNs, the opposite process is done. The input to the $\mathrm{NN}$ is the output from the physical system (percent drug release) while the output is the input to the system (US power and duty cycle). The inverse plant model is estimated and used to achieve model predictive control. Since there are many variations that can be used as the input (in this study we used the acoustic power and the pulsing behavior), the physical system produced multiple versions of output each corresponding to a different input. Unfortunately, it was noticed that the system behaved differently for each input which implies that a global plant model does not exist. Hence, an average model was adopted that produced the best performance when excited with all the inputs. There were 9 different variations of inputs that were used to produce 9 different plant models from which the average model was chosen. The outcome is discussed in details in the results section.

\section{RESULTS AND DISCUSSION}

Initially, the estimated liposomal drug delivery model was tested to ensure the accurate prediction of the output (release percent) for all variations of the input (US powers and duty cycles). As mentioned previously, 9 input-output relations were chosen to create 9 different models, from which an average approximate model was chosen to serve as the plant model. Table (1) shows the fitting percentages for the 9 different models under the name Actual Model. These models were tested against the data used to extract them. All models predicted the output with an accuracy of at least $83 \%$ which is 
an indication of the accuracy by which NNs were able to capture the complex non-linear behavior of the training data. A single liposomal drug delivery model is required in the MPC and this model is supposed to perform well when excited with various input US patterns. To find such a model, each of the derived 9 models was excited with a set of

9 different inputs and the fitting performance was calculated. This step guarantees choosing the best model performance for all inputs. Our simulation results showed that model 8 (average model 2) had the best average performance against all inputs. This model predicted the fitting with a $71.1 \%$ accuracy. It performed the worst when simulated with input 7 settings (3.88\% fitting) whereas, the best performance was achieved when simulated with input 5 settings $(92.73 \%$ fitting) as shown in the Table. In pursuit of a better performing model, another average model was constructed. This model was generated using a randomly generated average input. This average input was then passed through model 8 and the corresponding output recorded. Then using the average input and the corresponding output, an average model was found. The model was tested against the 9 inputs and it achieved $77.7 \%$ average fitting which is a better performance compared to the performance of model 8 . Nonetheless, even for this model, the accuracy was very low when excited with input 4 which can be considered an outlier. By excluding it from the accuracy calculation, the average fitting of this model is approximately $86.6 \%$. Therefore, this model was used as the liposomal drug delivery system for the MPC. Results are shown in Table 1 under Average Model 1. Similar procedures were followed to produce the inverse model, but using the drug release curves as the input while the US patterns as the output. Due to the edgy (step) nature of US patterns, the produced models were unstable. To solve this problem, moving average filtering was used to smooth the edges. The newly smoothed US patterns were used, and a stable inverse model was derived with an average accuracy of $72 \%$.

The motivation behind this work is to develop a model predictive controller capable of controlling the drug level in vitro so that the chemotherapeutic concentration is kept within a therapeutic window (in an attempt to prevent cancer cells from developing multi-drug resistance). This system can be used to envision the practical scenario below: Assume a medical doctor recommended using targeted chemotherapy via liposomes and ultrasound in the treatment of a cancer patient. The following conditions should also be met: (1) the sonication power should not exceed $10 \mathrm{~W}$ and (2) Drug Concertation Level (DCL) in the vicinity of the tumor should be stabilized at a certain level (e.g.100 mg/m2). Therefore, in a real-life clinical setting, liposomes will be injected into the patients body, US will be applied to cause drug release until the concentration level is at the required value. To fulfill the maximum intensity requirement, a release trajectory is defined such that US intensity is kept within the limits while increasing the concentration until the set point is reached. Once the set point is reached, the MPC should make sure that the concentration level is kept at that pre-set level. Figure 5 shows an example of such release trajectory that was fed to the designed MPC as its reference input. The results of the MPC moves are shown in Figures 6, 7, 8. These figures show the controller moves (US parameters), system output (drug concentration), and both the reference and the system output overlapped, respectively. As can be seen, the controller response is not exceeding the specified power of $10 \mathrm{~W}$. Furthermore, the controller is not giving clean pulses, but rather producing pulses mixed with ups and downs giving evidence that the developed MPC system is predicting the

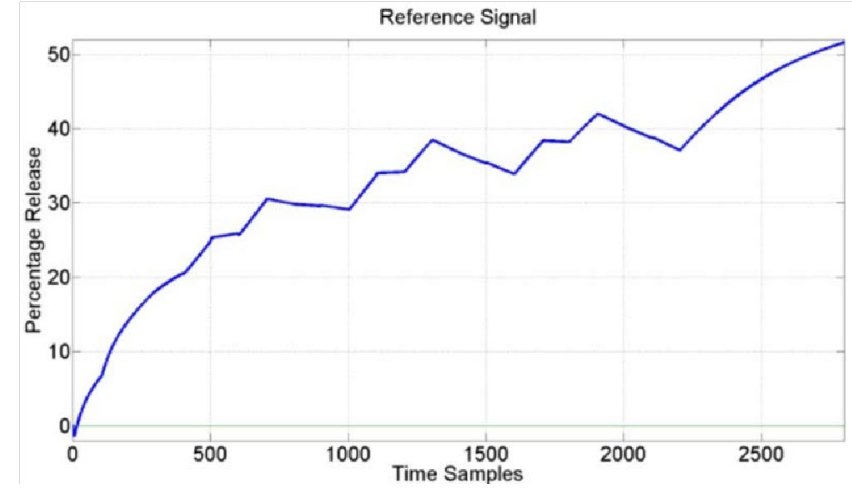

Fig. 5. Reference signal used as the set point or target in the controller.

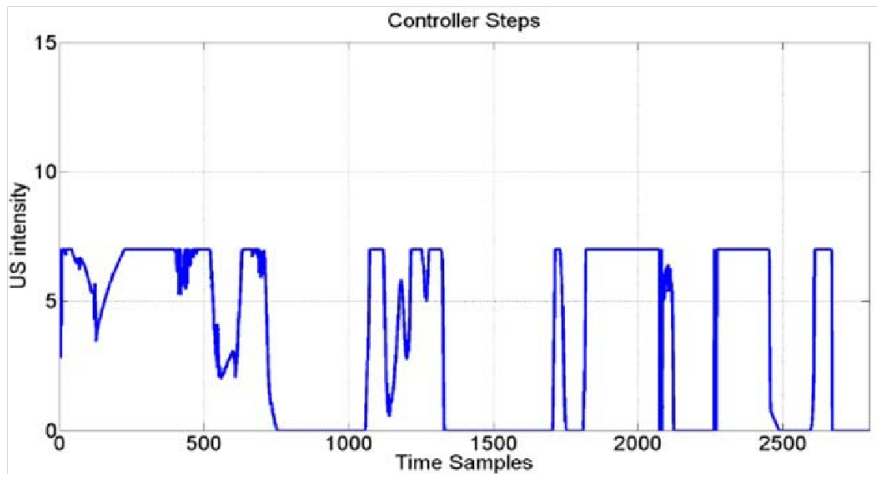

Fig. 6. Controller Moves versus time samples.

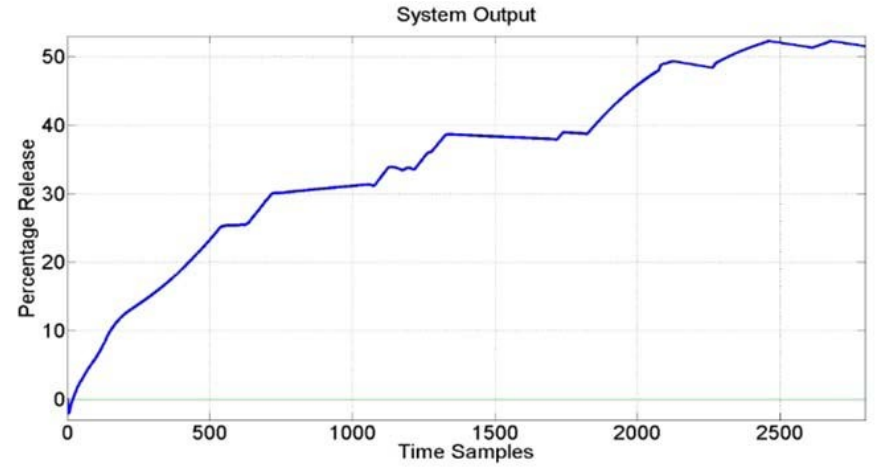

Fig. 7. System output based on the predicted controller moves vs time samples.

correct parameters necessary to keep the output on track with the reference. This is also evident in Figure 6 which compares the output with the references.

Therefore, as the results suggest, the developed MPC and the estimated average model are capable of following the reference 
signal and estimating the proper response of the model. The SSE difference between the output and the reference was calculated to be within an average of $5 \%$. This shows the robustness of the developed control system and its ability to control chemotherapeutic levels in vitro. It also shows that the model is stable and able to respond to a variable input.

The results of the system can be further improved by enhancing the model used in the MPC. Yet, in this work we present the concept of devising a control system capable of

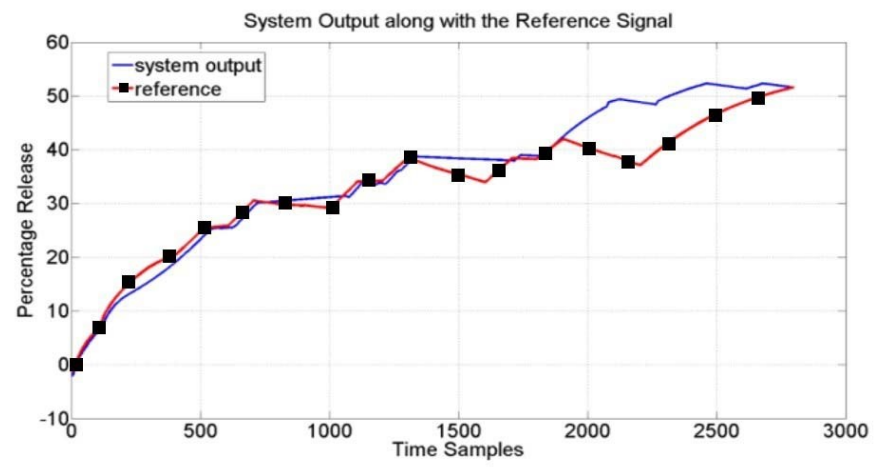

Fig. 8. Overlap between system output and the target curve.

keeping the drug concentration level at the tumor site at a prespecified level. This, in turn, lowers the risk of the patient developing multidrug resistance and may shorten the treatment period. Furthermore, as a part of future work recommendation, the experimental setup could be enhanced to accommodate more realistic and representative characteristics that closely mimic the real life scenario of liposomes injected into the body and US applied across the skin and actual feedback sensing mechanism to measure the actual drug concentration level. Furthermore, a proper way by which the drug concentration level can be accurately measured when dealing with in vivo cases shall be researched. The current mechanism suggested assumes the availability of devices capable of detecting the fluorescence level of the drugs through human tissue. However, in the future, other techniques might be proposed to serve this purpose.

\section{REFERENCES}

[1] J. D. O'Flaherty et al., "The cancer stem-cell hypothesis: Its emerging role in lung cancer biology and its relevance for future therapy," $J$. Thoracic Oncol., vol. 7, no. 12, pp. 1880-1890, Dec. 2012.

[2] V. Wiwanitkit, Advanced Nanomedicine and Nanobiotechnology. Hauppauge, NY, USA: Nova Science Publisher, 2008.

[3] Y. Liu, T.-S. Niu, L. Zhang, and J.-S. Yang, "Review on nano-drugs," Natural Sci., vol. 2, no. 1, pp. 41-48, 2010.

[4] T. L. Andresen, S. S. Jensen, and K. Jørgensen, "Advanced strategies in liposomal cancer therapy: Problems and prospects of active and tumor specific drug release," Progr. Lipid Res., vol. 44, no. 1, pp. 68-97, Jan. 2005.

[5] S. E. Ahmed, A. M. Martins, and G. A. Husseini, "The use of ultrasound to release chemotherapeutic drugs from micelles and liposomes," J. Drug Targeting, vol. 23, no. 1, pp. 16-42, 2015.

[6] D. Peer, J. M. Karp, S. Hong, O. C. Farokhzad, R. Margalit, and R. Langer, "Nanocarriers as an emerging platform for cancer therapy," Nature Nanotechnol., vol. 2, no. 12, pp. 751-760, 2007.
[7] S.-L. Huang and R. C. MacDonald, "Acoustically active liposomes for drug encapsulation and ultrasound-triggered release," Biochimica Biophys. Acta-Biomembranes, vol. 1665, nos. 1-2, pp. 134-141, Oct. 2004.

[8] K. Maruyama, O. Ishida, T. Takizawa, and K. Moribe, "Possibility of active targeting to tumor tissues with liposomes," Adv. Drug Del. Rev., vol. 40, nos. 1-2, pp. 89-102, 1999.

[9] H. Maeda, J. Wu, T. Sawa, Y. Matsumura, and K. Hori, "Tumor vascular permeability and the EPR effect in macromolecular therapeutics: A review," J. Controlled Release, vol. 65, nos. 1-2, pp. 271-284, Mar. 2000.

[10] H. Moussa, A. M. Martins, and G. A. Husseini, "Review on triggered liposomal drug delivery with a focus on ultrasound," Current Cancer Drug Targets, vol. 15, no. 4, pp. 282-313, May 2015.

[11] D. D. Lasic and D. Needham, "The 'Stealth' liposome: A prototypical biomaterial," Chem. Rev., vol. 95, no. 8, pp. 2601-2628, 1995.

[12] A. Akbarzadeh et al., "Liposome: Classification, preparation, and applications," Nanosc. Res. Lett., vol. 8, no. 1, pp. 1-9, 2013.

[13] N. H. Bergstrand, "Liposomes for drug delivery: From physicochemical studies to applications," Ph.D. dissertation, ProQuest, Ann Arbor, MI, USA, 2003.

[14] R. Murari, I. Ahmad, and S. S. Chrai, "Liposomes, part II: Drug delivery systems," Pharmaceutical Technol. Eur., vol. 15, p. 53, 2003.

[15] C. D. Landon, J.-Y. Park, D. Needham, and M. W. Dewhirst, "Nanoscale drug delivery and hyperthermia: The materials design and preclinical and clinical testing of low temperature-sensitive liposomes used in combination with mild hyperthermia in the treatment of local cancer," Open Nanomed. J., vol. 3, no. 1, pp. 38-64, Jan. 2011.

[16] F. Frézard, "Liposomes: From biophysics to the design of peptide vaccines," Brazilian J. Med. Biol. Res., vol. 32, no. 2, pp. 181-189, 1999.

[17] G. Gregoriadis and C. Davis, "Stability of liposomes in vivo and in vitro is promoted by their cholesterol content and the presence of blood cells," Biochem. Biophys. Res. Commun., vol. 89, no. 4, pp. 1287-1293, Aug. 1979.

[18] C. Kirby, J. Clarke, and G. Gregoriadis, "Cholesterol content of small unilamellar liposomes controls phospholipid loss to high density lipoproteins in the presence of serum," FEBS Lett., vol. 111, no. 3, pp. 324-328, 1980.

[19] Z. Li et al., "The ratio of phosphatidylcholine to phosphatidylethanolamine influences membrane integrity and steatohepatitis," Cell Metabolism, vol. 3, no. 5, pp. 321-331, May 2006.

[20] K. W. Ferrara, "Driving delivery vehicles with ultrasound," Adv. Drug Del. Rev., vol. 60, no. 10, pp. 1097-1102, Jun. 2008.

[21] H. M. Santos, C. Lodeiro, and J.-L. Capelo-Martínez, "The power of ultrasound," Ultrasound Chem., Anal. Appl., pp. 1-16, Nov. 2009.

[22] T. Nishita, "Heat-sensitive liposomes containing cisplatin and localized hyperthermia in treatment of murine tumor," Osaka City Med. J., vol. 44, no. 1, pp. 73-83, 1998.

[23] N. Saniei, "Hyperthermia and cancer treatment," Heat Transf. Eng., vol. 30, no. 12, pp. 915-917, 2009.

[24] G. F. Baronzio and E. D. Hager, Hyperthermia in Cancer Treatment: A Primer, Boston, MA, USA: Springer, 2006.

[25] P. Wust et al., "Hyperthermia in combined treatment of cancer," Lancet. Oncol., vol. 3, no. 8, pp. 487-497, 2002.

[26] L. Li et al., "Mild hyperthermia triggered doxorubicin release from optimized stealth thermosensitive liposomes improves intratumoral drug delivery and efficacy," J. Controlled Release, vol. 168, no. 2, pp. 142150, 2013.

[27] S. M. Park et al., "Novel temperature-triggered liposome with high stability: Formulation, in vitro evaluation, and in vivo study combined with high-intensity focused ultrasound (HIFU)," J. Controlled Release, vol. 170, no. 3, pp. 373-379, Sep. 2013.

[28] N. Y. Rapoport, A. M. Kennedy, J. E. Shea, C. L. Scaife, and K.-H. Nam, "Controlled and targeted tumor chemotherapy by ultrasound-activated nanoemulsions/microbubbles," J. Controlled Release, vol. 138, no. 3, pp. 268-276, Sep. 2009.

[29] S. K. Cool et al., "Coupling of drug containing liposomes to microbubbles improves ultrasound triggered drug delivery in mice," J. Controlled Release, vol. 172, no. 3, pp. 885-893, Dec. 2013. 
[30] C. C. Chen, P. S. Sheeran, S.-Y. Wu, O. O. Olumolade, P. A. Dayton, and E. E. Konofagou, "Targeted drug delivery with focused ultrasoundinduced blood-brain barrier opening using acousticallyactivated nanodroplets," J. Controlled Release, vol. 172, no. 3, pp. 795804, Dec. 2013.

[31] C. Oerlemans, R. Deckers, G. Storm, W. E. Hennink, and J. F. W. Nijsen, "Evidence for a new mechanism behind HIFU-triggered release from liposomes," J. Controlled Release, vol. 168, no. 3, pp. 327-333, Jun. 2013.

[32] J. R. Lattin, D. M. Belnap, and W. G. Pitt, "Formation of eLiposomes as a drug delivery vehicle," Colloids Surfaces B, Biointerfaces, vol. 89, pp. 93-100, Jan. 2012.

[33] J. R. Lattin, W. G. Pitt, D. M. Belnap, and G. A. Husseini, "Ultrasoundinduced calcein release from eLiposomes," Ultrasound Med. Biol., vol. 38, no. 12, pp. 2163-2173, Dec. 2012.

[34] D. E. Seborg, T. F. Edgar, and D. A. Mellichamp, "Model predictive control," in Process Dynamics and Control, 2nd ed., B. Zobrist, Ed. Hoboken, NJ, USA: Wiley, 2004, pp. 534-565.

[35] K. Patan, "Neural network-based model predictive control: Fault tolerance and stability," IEEE Trans. Control Syst. Technol., vol. 23, no. 3, pp. 1147-1155, May 2015.

[36] L. Magni, D. M. Raimondo, and F. Allgower, "Nonlinear model predictive control: Theories and algorithms," in Communications and Control Engineering. New York, NY, USA: Springer, 2009.

[37] G. A. Husseini, N. M. Abdel-Jabbar, F. S. Mjalli, W. G. Pitt, "Modeling and sensitivity analysis of acoustic release of Doxorubicin from unstabilized pluronic P105 using an artificial neural network model," Technol. Cancer Res. Treatment, vol. 6, no. 1, pp. 49-56, 2007.

[38] G. A. Husseini, F. S. Mjalli, W. G. Pitt, and N. M. Abdel-Jabbar, "Using artificial neural networks and model predictive control to optimize acoustically assisted Doxorubicin release from polymeric micelles," Technol. Cancer Res. Treatment, vol. 8, no. 6, pp. 479-488, 2009.

[39] H.-Y. Lin and J. L. Thomas, "PEG-lipids and oligo(ethylene glycol) surfactants enhance the ultrasonic permeabilizability of liposomes," Langmuir, vol. 19, no. 4, pp. 1098-1105, 2003.

[40] T. Day and A. F. Read, "Does high-dose antimicrobial chemotherapy prevent the evolution of resistance?" PLoS Comput. Biol., vol. 12, no. 1, p. e1004689, 2016.

[41] S. E. Ahmed, H. G. Moussa, A. M. Martins, M. H. Al-Sayah, and G. A. Husseini, "Effect of $\mathrm{pH}$, ultrasound frequency and power density on the release of calcein from stealth liposomes," Eur. J. Nanomed., vol. 8, no. 1, pp. 31-43, 2016. 